\title{
One-reason decision-making: Modeling violations of expected utility theory
}

\author{
Konstantinos V. Katsikopoulos • Gerd Gigerenzer
}

Published online: 17 June 2008

(C) Springer Science + Business Media, LLC 2008

\begin{abstract}
People violate expected utility theory and this has been traditionally modeled by augmenting its weight-and-add framework by nonlinear transformations of values and probabilities. Yet individuals often use one-reason decision-making when making court decisions or choosing cellular phones, and institutions do the same when creating rules for traffic safety or fair play in sports. We analyze a model of one-reason decision-making, the priority heuristic, and show that it simultaneously implies common consequence effects, common ratio effects, reflection effects, and the fourfold pattern of risk attitude. The preferences represented by the priority heuristic satisfy some standard axioms. This work may provide the basis for a new look at bounded rationality.
\end{abstract}

Keywords Decision making $\cdot$ EVT $\cdot$ EUT $\cdot$ St. Petersburg Paradox

Most descriptive theories of decision-making - and certainly those that are variants of expected value theory (EVT) or expected utility theory (EUT) - make the following psychological assumptions:

1. Independent evaluations: Every option has a value that is measured by a single number (options are not evaluated relative to other options).

2. Exhaustive search: The value of an option is calculated by using all available information (for gambles, the probabilities and values for all possible outcomes).

3. Trade-offs: To calculate an option's value, low values on one attribute (e.g., a value) can be compensated by high values on another attribute (e.g., a probability).

EVT makes two additional assumptions for calculating an option's value:

4. Objective probabilities: The probabilities used to calculate an option's value are equal to the objective probabilities (the objective probabilities are not transformed in a nonlinear way).

K. V. Katsikopoulos $(\bowtie) \cdot$ G. Gigerenzer

Center for Adaptive Behavior and Cognition, Max Planck Institute for Human Development,

Lentzeallee 94, 14195 Berlin, Germany

e-mail: katsikop@mpib-berlin.mpg.de 
5. Objective values: The outcome values used to calculate an option's value are equal to the objective monetary values (the objective values are not transformed in a nonlinear way).

To account for the St. Petersburg Paradox (Jorland 1987), which violates EVT, Daniel Bernoulli (1738) introduced EUT, which changes the objective values assumption 5. To account for further findings such as buying lottery tickets and insurance policies, the Allais paradox (Allais 1979), as well as the fourfold pattern of risk attitude (Tversky and Kahneman 1992), modifications of EUT relax assumptions 4 and 5 by using nonlinear transformations with a number of free parameters.

In this paper we follow Selten's (2001) and others' call to develop and analyze a fundamental alternative approach to modeling choice. Specifically, we target the basic assumptions 1 to 3 but retain assumptions 4 and 5. In Section 1, we briefly review some of the empirical evidence for simple heuristics that do not retain assumptions 1 to 3 . These heuristics are a way of implementing Simon's bounded rationality $(1955,1956)$, using the approach of Gigerenzer and Selten (2001), Leland (1994), and Rubinstein (1988).

In Section 2, we present the priority heuristic, proposed by Brandstätter et al. (2006), which is related to lexicographic semi-orders (Luce 1956; Tversky 1969), and predicted empirical data better than modifications of EUT such as cumulative prospect theory did. In that paper, specific examples were used to numerically illustrate that the priority heuristic can account for major violations of expected utility theory (such as the Allais paradox and the fourfold pattern of risk attitude). But no mathematical conditions were derived under which the priority heuristic predicts these violations. In Section 3, we prove under which conditions the heuristic implies major violations of EUT. This facilitates understanding how the Allais paradox, the fourfold pattern of risk attitude, and other systematic patterns, may arise from cognitive processes that implement one-reason decision-making, rather than from probability weighting and utility functions.

For further understanding of the heuristic, in Section 4, we derive conditions under which the preferences represented by the heuristic satisfy standard axioms. In Section 5, we discuss the relation of the priority heuristic to Rubinstein's (1988) similarity model. We conclude that simple heuristics that make no trade-offs seem to be a promising approach to modeling bounded rationality.

\section{Why simple heuristics?}

Assumptions 1 to 3 are commonplace. Why do we propose they be reconsidered? In this section, we discuss two reasons: empirical evidence for people's use of heuristics that violate these assumptions and prescriptive reasons for why these heuristics can make quick and accurate predictions.

\subsection{Empirical evidence}

People often do not evaluate options in isolation but instead relative to at least one other option. For instance, when judging the value or size of objects, ratings are 严 Springer 
more inconsistent - both within and between individuals - when objects are evaluated independently rather than in comparison to other objects (e.g., Gigerenzer and Richter 1990). Different choices are made depending on the other options in the choice set (Shafir et al. 1993) and on the other options preceding an option when these are sequentially presented (Schwarz 1999; Schwarz et al. 1985). Regret theory (Loomes and Sugden 1987) and range-frequency theory (Parducci 1965) both model the relative evaluation of options. Based on this and other evidence, Luce and von Winterfeldt (1994, p. 267) conclude that "no theory that is based on separate evaluations of gambles can possibly work." Thus, psychologically, a class of situations exists in which an option has a value only relative to other options.

The second assumption is that the value of an option is calculated by searching for all available information. This assumption is unrealistic in many contexts, such as on the Internet, where there is too much information and limited search is necessary. Similarly, experimental research has shown that people do not search exhaustively but employ limited search, both in internal search (in memory) and in external search (e.g., in libraries; Payne et al. 1993). A number of theories have modeled limited search, both within the framework of optimization (Stigler 1961) and satisfying (Simon 1955, 1956). In the extreme, search could terminate after the first reason that allows for a decision, thus making no trade-offs. Bröder (2000, 2003; Bröder and Schiffer 2003) report that under various conditions (e.g., time pressure, high information costs) a majority of people rely on lexicographic heuristics that look up one reason at a time and stop as soon as a reason allows them to do so. Rieskamp and Otto (2006) and Rieskamp and Hoffrage (1999) show that people adapt the length of search to the structure of the problem.

Third, the experimental evidence shows that people often do not make trade-offs but base their decisions on heuristics that are "non-compensatory," which means that low values on one attribute (value or probability) cannot be compensated by high values on others. These no-trade-off heuristics include lexicographic models, conjunctive rules, disjunctive rules, and elimination-by-aspects (see also Lilly 1994). Consider this classic review of 45 studies in which the process of decisionmaking was investigated by means of Mouselab, eye movement, and other process tracking techniques (Ford et al. 1989). Varying between studies, the choices to be made included apartments, microwaves, and birth control methods:

"The results firmly demonstrate that non-compensatory strategies were the dominant mode used by decision makers. Compensatory strategies were typically used only when the number of alternatives and dimensions were small or after a number of alternatives have been eliminated from consideration.” (p. 75).

Consistent with this result, most subsequent studies that reported trade-offs have used only a small number of attributes (typically only 2 to 4 ) and have fitted the data by means of conjoint analysis or other linear models without testing lexicographic or other no-trade-off models. Studies that investigated consumer choice on the Internet and in other situations with large numbers of alternatives and cues - and that tested models with stopping rules - concluded that a majority of participants followed noncompensatory processes. For instance, Yee et al. (2007) reported that when people had a choice between 32 SmartPhones that varied on six cues, non-compensatory models predicted their choices better than Bayesian and other models that assume 
trade-offs did. Similarly, when people chose between cameras varying on seven attributes with two to six levels each, non-compensatory strategies again provided the best prediction: $58 \%$ relied on one attribute only, $33 \%$ relied on two attributes, and only $2 \%$ used three attributes (Gilbride and Allenby 2004). Experiments in which participants chose a product (such as an answering machine or toaster) from the Web sites CompareNet and Jango showed the same result: The larger the number of alternatives offered, the more customers relied on a no-trade-off strategy (Jedetski et al. 2002). Bröder and his colleagues (Bröder 2000; Bröder and Schiffer 2003) conducted 20 studies, concluding that a lexicographic heuristic, Take The Best, is used under a number of conditions such as when information is costly and the variability of the validity of the attributes is high. Bröder and Gaissmaier (2007) and Nosofsky and Bergert (2007) showed that Take The Best predicts response times better than weighted additive and exemplar models. Thus, the experimental evidence strongly suggests that heuristics that rely on limited search and do not make tradeoffs are in people's "adaptive toolbox" (Gigerenzer and Selten 2001), and that these heuristics are selected in a sensitive way according to the structure of the problem (Gigerenzer et al. 1999; Lopes 1995; Payne et al. 1993).

\subsection{Prescriptive reasons}

The empirical evidence cited above shows that people proceed differently from assumptions 1 to 3 . Relying on limited search and foregoing trade-offs, however, does not generally imply that these decisions are inferior or irrational. First, institutions routinely apply lexicographic rules in designing environments in order to make them safer and more transparent and allow human minds to operate in an efficient way. Which vehicle has the right of way at a crossing is defined by a lexicographic rule, not by a trade-off between the police officer's hand signal, the color of the traffic light, the traffic sign, and where the other car is coming from. Similarly, in soccer and hockey, the national and international associations agreed on lexicographic rules to determine the final standing within a group of competing teams. The Arabic number system allows using a simple lexicographic rule to decide which of two numbers is larger, employing order and limited search unavailable in other systems.

Second, lexicographic heuristics can also be accurate. Under certain conditions, they are more accurate than multiple regression and other linear models that make tradeoffs (Gigerenzer et al. 1999; Martignon and Hoffrage 2002; Hogarth and Karelaia 2005, 2006; Baucells et al. 2008), as well as nonlinear models such as neural networks and classification and regression trees (Brighton 2006). Lexicographic heuristics can even be optimal (Katsikopoulos and Martignon 2006). We would like to emphasize these results, given that ever since lexicographic rules were first proposed in economics by Carl Menger, decision researchers have often dismissed them as a form of irrationality (see also Fishburn 1974). But how can it be that heuristics are accurate?

In fact, there are good mathematical reasons for their accuracy. First, the heuristics tend to be robust. That is, they do not lose much of their accuracy between fitting known data and predicting new data. In contrast, models with numerous free parameters tend to over-fit the data and lose accuracy in prediction (Roberts and 记 Springer 
Pashler 2000). Second, lexicographic heuristics can exploit a number of structural properties, such as the presence of cumulatively dominating options in the choice set (Baucells et al. 2008) or large differences in the statistical informativeness of attributes (Martignon and Hoffrage 2002; Hogarth and Karelaia 2005, 2006; Katsikopoulos and Fasolo 2006; Katsikopoulos and Martignon 2006). Simulation studies have shown that these properties are relatively common.

A major unresolved problem in the tradition of revising EVT and EUT by using free parameters is that none of the estimated sets of parameters in models such as cumulative prospect theory can simultaneously account for buying lottery tickets, buying insurance policies, the Allais paradox, and other choice patterns observed in the literature (Neilson and Stowe 2002). For instance, the functions estimated by Camerer and Ho (1994) and $\mathrm{Wu}$ and Gonzalez (1996) imply that people will purchase neither lottery tickets nor insurance policies. Moreover, Neilson and Stowe (2002) concluded that the troubles run deeper; they showed that no parameter combinations allow for these two behaviors and a series of choices made by a large majority of participants and reasonable risk premia. Similarly, Blavatskyy (2005) showed that the conventional parameterizations of cumulative prospect theory do not explain the St. Petersburg paradox. Overall, the parameter values fitted to one set of data are unlikely to be robust, in the sense of generating accurate predictions for new sets of data. On the other hand, simple heuristics such as the priority heuristic have no free parameters and tend to be robust (Gigerenzer et al. 1999; Martignon and Hoffrage 2002).

\section{The priority heuristic}

The priority heuristic specifies how people chose between two gambles by (1) evaluating the gambles in relation to each other (as opposed to independently), (2) relying on limited search (as opposed to using all information), and (3) without making trade-offs between attributes (values and probabilities). The priority heuristic specifies how values and probabilities are ordered according to a priority rule, how a stopping rule terminates the search for information, and how a decision is made based on the available information. Unlike the priority heuristic, prospect theory and other modifications of EUT assume exhaustive search and they have no stopping rules and thus the order of attributes is assumed to be of no importance. The heuristic does not need nonlinear transformations of value and probability but takes attributes in their natural currencies (i.e., it uses objective values and objective probabilities). Finally, the priority heuristic does not have any free parameters.

For a couple of two-outcome gambles with non-negative values (hereafter referred to as gains), the priority heuristic implements the following sequential process:

Priority rule Go through attributes in the order: minimum gain, probability of minimum gain, maximum gain.

Stopping rule Stop information search if the minimum gains differ by one tenth (or more) of the maximum gain (across the two gambles); otherwise, stop search if probabilities of minimum gain differ by 0.1 (or more). 
Decision rule Choose the gamble that is more attractive in the attribute (gain or probability) that stopped search.

We will refer to the one tenth of the maximum gain as the aspiration level for gains and to 0.1 as that for probabilities. ${ }^{1}$ The more attractive gamble is the one with the higher (minimum or maximum) gain or with the lower probability of minimum gain. The first step of the priority heuristic - comparing minimum gains - is identical to the minimax heuristic except that there is an aspiration level that determines whether minimax is followed or not. The last step - comparing maximum gains - is identical to the maximax heuristic.

For non-positive values (hereafter called losses), the difference is that "gain" is replaced by "loss". The more attractive loss is the lower one and the more attractive probability of minimum loss is the higher one. In this paper, we do not consider gambles that obtain both gains and losses. If the gambles have more than two outcomes, the probability of the maximum gain (or loss) is the fourth attribute. If no attribute leads to a prediction, a gamble is picked randomly. The heuristic applies to "difficult" choices, that is, non-dominated gambles and gambles where the expected values are relatively close (see below). If instead one alternative dominates the other, or the expected values are far apart, the assumption is that choice is made without going through the process ${ }^{2}$.

We now explain in more detail how the parameters of the priority heuristic were set a-priori according to empirical evidence and logical constraints. The priority heuristic has three fixed parameters: the order of attributes and the two aspiration levels for values and probabilities. Consider the order of attributes first, using the example of two-outcome gambles with non-negative values. A simple gamble with two outcomes has four attributes: minimum and maximum gains, and their respective probabilities. This amounts to 24 possible orderings. The logical constraint that the two probabilities are complementary reduces the number of attributes to three, and the number of orderings to six. Empirical evidence suggests that values are more important than probabilities (for a review, see Brandstätter et al. 2006). For instance, insurance buyers focus on the potential large loss rather than on the low probabilities; lottery ticket buyers focus on the big gains rather than small probabilities (Daston 1988); emotional outcomes tend to override the impact of probabilities (Sunstein 2003); and in the extreme, people neglect probabilities altogether and base their choices on the immediate feeling elicited by the gravity or benefits of future events (Loewenstein et al. 2001). If gains are the first consideration, this reduces the possible number of orders from six to four. But which gain is considered first, the minimum or the maximum gain? The empirical evidence seems to favor the minimum gain, consistent with the motivation to avoid the worst outcome and to avoid failure (Heckhausen 1991). This further reduces the

\footnotetext{
${ }^{1}$ When we use the term "nonlinear" transformation in this paper, we refer to continuous functions as in the probability weighting function of prospect theory, not to a simple step-function as represented by the aspiration levels. For simplicity, we do not deal here with the idea that aspiration levels for values are rounded to the closest prominent number (Brandstätter et al. 2006, p. 413).

${ }^{2}$ If the expected values are relatively far apart (by a factor of more than 2; see below), people can often "see" that the choice is obvious. Similarly, one can see that $71 \times 11$ is larger than $18 \times 13$ without calculating the products. There are probably a number of simple heuristics that help us to see which is larger, such as Rubinstein's similarity rule (see Section 5) and cancellation: If two numbers are close, ignore them, and compare the other two numbers. In the example 11 and 13 would be ignored.
} 
number of possible orders to two. Experiments indicate that for most people, the probability of the minimum gain is ranked second (Brandstätter et al. 2006; Slovic, Griffin and Tversky 1990, study 5). This results in the order: minimum gain, probability of minimum gain, maximum gain.

Which difference in minimum gains is large enough to stop the process and choose the gamble with the higher minimum gain? The existence and operation of aspiration levels has been long demonstrated in choice (Lopes 1995). Empirical evidence suggests that this aspiration level is not fixed but increases with the maximum gain, and it is defined by our cultural base-10 system (Albers 2001). This leads to the hypothesis that the aspiration level is one tenth of the maximum gain. Furthermore, unlike the value scale, which has open ends, the probability scale is bounded at both ends. There are several reasons why the aspiration level for probabilities has to be a difference rather than a ratio (Leland 2002). The most important is that probabilities, unlike gains, have complements, so that an increase from 0.10 to 0.15 is equivalent to a decrease from 0.90 to 0.85 ; this point is captured by differences but not by ratios. A simple hypothesis is that one tenth of the probability scale, that is, 10 percentage points difference, is large enough. Empirical evidence suggests that people typically do not make more fine-grained differences except at the ends of the probability scale (Albers 2001).

This is not to say that other parameter values would be impossible; given the consistent report of individual differences, they might in fact explain some of these. As the remainder of the paper will make clear, however, the intent is to show that a lexicographic model based on crude estimates of order and aspiration levels, that has been successful in predicting people's choice of gambles, can be shown to imply major "anomalies" in choice.

\subsection{Predictive power}

To illustrate how the heuristic works, consider one of the problems posed by Allais (1979), known as the Allais paradox, where people choose first between gambles $A$ and $B$ :

A: $100,000,000$ with probability 1.00

B: $500,000,000$ with probability 0.10

$100,000,000$ with probability 0.89

$0 \quad$ with probability 0.01

By subtracting a 0.89 probability to win 100 million from both gambles $A$ and $B$, Allais obtained the following gambles, $C$ and $D$ :

C: $100,000,000$ with probability 0.11

$0 \quad$ with probability 0.89

D: $500,000,000$ with probability 0.10

$0 \quad$ with probability 0.90

The majority of people chose gamble $A$ over $B$ and $D$ over $C$ (MacCrimmon 1968 ) which constitutes a violation of the independence axiom of EUT (see below). EUT does not predict whether $A$ or $B$ will be chosen; it only makes conditional predictions such as "if $A$ is chosen, then $C$ is chosen." The priority heuristic, in 
contrast, makes stronger predictions: It predicts whether $A$ or $B$ will be chosen, and whether $C$ or $D$ will be chosen. Consider the choice between $A$ and $B$. The maximum gain is 500 million and therefore the aspiration level for gains is 50 million. The difference between the minimum gains equals $(100-0)=100$ million that exceeds the aspiration level, and search is stopped. The gamble with the more attractive minimum gain is $A$. Thus the heuristic predicts the majority choice correctly.

In the choice between $C$ and $D$, minimum gains are equal. Thus the next attribute is looked up. The difference between the probabilities of minimum gains equals $0.90-$ $0.89=0.01$ which is smaller than the aspiration level for probabilities of 0.1 . Thus the choice is decided by the last attribute, maximum gain, in which gamble $D$ is more attractive. This prediction is again consistent with the choice of the majority. Thus the priority heuristic predicts the Allais paradox.

How well does this simple heuristic overall explain people's choices? Brandstätter et al. (2006) compared three modifications of EUT — cumulative prospect theory (Tversky and Kahneman 1992), security-potential/aspiration theory (Lopes and Oden 1999), and transfer-of-attention-exchange model (Birnbaum and Chavez 1997) - with the priority heuristic on how well they could predict the majority choice in four published sets of gambles. The sets were from Kahneman and Tversky (1979), Tversky and Kahneman (1992), Lopes and Oden (1999), and Erev et al. (2002) and included various kinds of gambles: two-outcome gambles, choices based on certainty equivalence, five-outcome gambles, and randomly generated gambles. Across the 260 pairs of gambles, the priority heuristic predicted $87 \%$ of the choices correctly, followed by $79 \%, 77 \%$, and $69 \%$ for security-potential/aspiration theory, cumulative prospect theory, and transferof-attention-exchange model, respectively. Ten other heuristics (including minimax and maximax) were also tested and all performed less well, with many at chance level.

The limits of the predictive power of the priority heuristic were analyzed using a set of 450 pairs of gambles. The priority heuristic was more predictive than the modifications of EUT when the problems were difficult in the sense that the expected values of the two gambles are close, with a ratio of at most 2 . When the problems were easy (ratio is larger than 2), the modifications of EUT did better than the priority heuristic, but none of them could outperform EVT. Both the priority heuristic and EVT use objective monetary values and probabilities, and no modifications of EUT that uses nonlinear transformations were more predictive. This raises the possibility that objective values and probabilities may suffice for predicting people's choices.

In summary, the first limit of the priority heuristic is that it does not account better than EVT and modifications of EUT for easy problems. The second limit is that it does not model individual differences (unless one introduces free parameters for order and aspiration levels that, however, should be measured independently rather than fitted to the data). We now show that this simple heuristic, even without free parameters, implies major violations of EUT.

\section{Explaining violations of EUT}

For the remainder of this article, we denote values by $x, y, \ldots$, probabilities by $p, p^{\prime}, \ldots$, and gambles by $G, G^{\prime}, \ldots$ Gambles are also symbolized explicitly, for Springer 
example, for a simple gamble, $(x, p ; 0,1-p)$. It is assumed that probabilities do not equal 0 or 1 . A gamble where $x$ occurs with probability 1 is denoted by $(x, 1)$. Compound gambles are symbolized by $\left(G, p ; G^{\prime}, 1-p\right)$. For our analyses, we assume that compound gambles are equivalent to their reduced simple-gamble form. This, however, does not mean that we also assume that people obey accounting axioms.

The relation "> ${ }_{h}$ " on the space of pairs of gambles is defined as follows: $G>_{h} G$ ' if and only if the priority heuristic predicts that $G$ is chosen over $G^{\prime}$ without using a random device. If the heuristic has to use such a device, we write $G={ }_{h} G^{\prime}$. If $G>_{h} G^{\prime}$ or $G={ }_{h} G^{\prime}$, we write $G \geq_{h} G^{\prime}$. To simplify, in the remainder of this article, we will use a version of the priority heuristic in which search is not stopped if the value and probability differences are equal to their respective aspiration levels. For example, if the probabilities of minimum gain equal 0.35 and 0.25 , their difference, 0.10 , is not large enough to stop search.

A core axiom of EUT is the independence axiom: for all gambles $G, G^{\prime}, G^{\prime \prime}$ and for all $p$, if $G$ is preferred to $G^{\prime}$, then $\left(G, p ; G^{\prime \prime}, 1-p\right)$ is preferred to $\left(G^{\prime}, p ; G^{\prime \prime}, 1-p\right)$. Two kinds of empirical findings, common consequence effects and common ratio effects, violate the independence axiom (Starmer 2000). ${ }^{3}$ We start this section by characterizing the conditions on values and probabilities under which the priority heuristic predicts these effects.

The priority heuristic predicts common consequence (CC) effects if the following statement holds:

$$
\begin{aligned}
& (y, 1)>_{h}(G, p ; y, 1-p) \text { and }(G, p ; 0,1-p)>_{h}(y, p ; 0,1-p), \\
& \qquad \text { where } G=\left(x, p^{\prime} ; 0,1-p^{\prime}\right) \text { and } x>y>0 .
\end{aligned}
$$

The common consequence is $y$ in the first pair of gambles and 0 in the second pair of gambles (occurring, in all cases, with probability $1-p$ ). In the Allais paradox ${ }^{4}, y=$ $100,000,000, p=0.11, x=500,000,000$, and $p^{\prime}=10 / 11$.

Result 1 (common consequence) Statement (CC) holds if and only if $y / x>0.1>p\left(1-p^{\prime}\right)$

Proof The reduced form of $(G, p ; y, 1-p)$ is $\left(x, p p^{\prime} y, 1-p ; 0, p\left(1-p^{\prime}\right)\right)$. It is clear that $(y, 1)>_{h}\left(x, p p^{\prime} ; y, 1-p ; 0, p\left(1-p^{\prime}\right)\right)$ if and only if minimum gain stops search, that is, if and only if $y-0>(0.1) x$, or $y / x>0.1$.

\footnotetext{
${ }^{3}$ Because we have assumed that the reduction of compound gambles holds, violations of independence come from violations of so-called preference axioms such as consequence monotonicity (Luce 1990).

${ }^{4}$ Another approach to studying the Allais paradox is to decompose it into axioms so that, if they all hold, the paradox disappears. Birnbaum (2004) identified three such axioms: transitivity, restricted branch independence, and coalescing. We derive conditions under which the priority heuristic predicts transitivity in the next section. It is possible to similarly study restricted branch independence - which is a weaker version of Savage's (1954) "sure thing" axiom - but the conditions become complicated and we do not report them here. Coalescing states that in a gamble, with two outcomes that yield the same value with the same probability, the two outcomes can be combined into a single one. Just as we assumed that, for our analyses, reduction of compound gambles holds, we also assume that coalescing holds.
} 
The reduced form of $(G, p ; 0,1-p)$ is $\left(x, p p^{\prime} ; 0,1-p p^{\prime}\right)$. It holds that $\left(x, p p^{\prime} ; 0,1-p p^{\prime}\right)>_{h}(y, p ; 0,1-p)$ if and only if the probability of minimum gain does not stop search, that is, if and only if $\left(1-p p^{\prime}\right)-(1-p)<0.1$, or $p(1-p)<0.1$.

Remark 1 In words, the priority heuristic implies common consequence effects whenever the minimum (non-zero) gain (of all gains in CC) is larger than one-tenth of the maximum gain and the probability of a zero gain for the risky option in the first pair of gambles (in CC) is smaller than one tenth. These factors of one-tenth correspond to the aspiration levels in the priority heuristic. The condition in Result 1 is fulfilled in the Allais paradox: $y / x=0.2$ and $p\left(1-p^{\prime}\right)=0.01$.

Common ratio effects can be modeled as follows (Starmer 2000): people's choice between $(x, \lambda p ; 0,1-\lambda p)$ and $(y, p ; 0,1-p)$ where $x>y>0$ and $\lambda>0$ changes as $p$ changes $(x, y$, and $\lambda$ are held constant). For example, consider the following choice problem:

$\begin{array}{ll}\text { A: } & 6,000 \text { with probability } 0.45 \\ 0 & \text { with probability } 0.55\end{array}$

B: $\quad 3,000$ with probability 0.90

$0 \quad$ with probability 0.10

Kahneman and Tversky (1979) found that the majority of people (86\%) chose gamble $B$. Now consider a second problem:

C: $\quad 6,000$ with probability 0.001

$0 \quad$ with probability 0.999

D: $\quad 3,000$ with probability 0.002

$0 \quad$ with probability 0.998

Here most people (73\%) chose gamble $C$. This is a common ratio effect with $x=$ $6,000, y=3,000$ and $\lambda=1 / 2$. Note that the gambles have equal expected values (this need not be the case for all common ratio problems). This finding is referred to as a "possibility" effect to emphasize that choices change as gains change, from probable in the $A$-or- $B$ problem, to merely possible in the $C$-or- $D$ problem. The priority heuristic predicts that gamble $B$ is chosen because minimum gains are equal (0) while probability of minimum gain stops search $(0.55-0.10>0.10)$ and $B$ is more attractive in it. The priority heuristic predicts that $C$ is chosen because it is more attractive in maximum gain.

The priority heuristic predicts common ratio (CR) effects for gambles with equal expected values if the following statement holds:

$$
\begin{gathered}
(y, p ; 0,1-p)>_{h}(x, \lambda p ; 0,1-\lambda p) \text { and }(x, \lambda c p ; 0,1-\lambda c p)>_{h}(y, c p ; 0,1-c p), \\
\text { where } x>y>0, \lambda=y / x, c>0 .
\end{gathered}
$$

In the example above, $c=1 / 450$.

In both problems in (CR) the gamble with lower maximum gain $(y)$ is chosen if and only if search stops by the probability of minimum gain. This follows because the minimum gains are always equal to zero and do not stop search. Computing the 
differences between the probabilities of minimum gain and rearranging terms, we have:

Result 2 (common ratio) Statement (CR) holds if and only if $(1-\lambda) p>$ $0.1>(1-\lambda) c p$.

Remark 2 In words, the priority heuristic implies common ratio effects (for gambles with equal expected values) if and only if the difference between the probabilities of minimum (zero) gain in the first pair of gambles (in CR) is larger than one-tenth and the difference between the probabilities of minimum gain (zero) in the second pair of gambles is smaller than one tenth. This one tenth corresponds to the aspiration level for probabilities in the priority heuristic.

To model the next violation of EUT, we define, for any gamble $G$, its opposite $-G$ to be the gamble that obtains value $-x$ when $G$ obtains $x$ (and with the same probability). For example, if $G=(5,0.6 ; 0,0.4)$, then $-G=(-5,0.6 ; 0,0.4)$.

Kahneman and Tversky (1979) found that choices are reversed when gambles are substituted by their opposites - these are called reflection effects. For example, $80 \%$ of their participants chose $(3,000,1)$ over $(4,000,0.8 ; 0,0.2)$ but $92 \%$ chose $(-4,000,0.8 ; 0,0.2)$ over $(-3,000,1)$. The priority heuristic predicts the choice of $(3,000,1)$ based on minimum gain and the choice of $(-4,000,0.8 ; 0$, 0.2 ) based on minimum loss. More generally, it is easy to see that the following holds:

Result 3 (reflection) $G>{ }_{h} G^{\prime}$ if and only if $-G^{\prime}>_{h}-G$.

Remark 3 Because the priority heuristic uses the same process for both gains and losses, it considers minimum gains and minimum losses first, respectively. Prioritizing minimum gains reflects the motivation to avoid the worst, while prioritizing minimum losses reflects the motivation to seek the best. The same reasoning applies to the other attributes, resulting in reflection effects.

The final violation of EUT we consider is the fourfold pattern of risk attitude (for comparing a gamble with one outcome to a gamble with two outcomes; Tversky and Kahneman 1992). This finding challenges the assumption of universal risk aversion and expresses the purchase of both lottery tickets and insurance policies. People were found to be risk taking for losses: for example, Tversky and Fox (1995) found that people's median certainty equivalent $(\mathrm{CE})$ for $(-100,0.95 ; 0,0.05)$ equals -84 . But Tversky and Fox (1995) also found that people are risk taking for gains if the gain probability is low-the CE of $(100,0.05 ; 0,0.95)$ was 14 . Conversely, people were found to be risk averse for high-probability gains such as $(100,0.95 ; 0,0.05$; $\mathrm{CE}=78)$ and for low-probability losses such as $(-100,0.05 ; 0,0.95 ; \mathrm{CE}=-8)$.

The priority heuristic can predict this pattern. Result 4 below refers to the case where the risky gamble has one non-zero outcome and Result 5 is the generalization for two non-zero outcomes.

Result 4 (Fourfold Pattern) Let $x>0$. It holds that (i) $(p x, 1)>_{h}(x, p ; 0,1-p)$ if and only if $p>0.1$, and (ii) $(-x, p ; 0,1-p)>_{h}(-p x, 1)$ if and only if $p>0.1$. 
Proof 1. If $p>0.1$, minimum gain stops search because $p x-0=p x>(0.1) x$. Thus $(x p, 1)>_{h}(x, p ; 0,1-p)$. If $p \leq 0.1$, probability of minimum gain is also looked up but it does not stop search. The gamble $(x, p ; 0,1-p)$ has more attractive maximum gain than $(p x, 1)$, thus $(x, p ; 0,1-p)>_{h}$ $(x p, 1)$.

2. It suffices to combine (i) with Result 3.

Result 5 (Fourfold Pattern) Let $x>y>0, z=p x+(1-p) y$, and $p^{*}=(0.1) x /$ $(x-y)$. It holds that (i) $(z, 1)>_{h}(x, p ; y, 1-p)$ if and only if $p>p^{*}$, and (ii) $(-x, p ;-y$, $1-p)>_{h}(-z, 1)$ if and only if $p>p^{*}$.

Proof 1. If $p>p^{*}$, minimum gain stops search because $z-y=p x+(1-p) y-y=$ $p(x-y)>p^{*}(x-y)=(0.1) x$. Thus $(z, 1)>_{h}(x, p ; y, 1-p)$. If $p \leq p^{*}$, minimum gain does not stop search. The gamble $(x, p ; y, 1-p)$ has more attractive probability of minimum gain as well as maximum gain than $(z, 1)$. Thus, $(x, p ; y, 1-p)>_{h}(z, 1)$.

2. It suffices to combine (i) with Result 3.

Remark 4 These proofs show that the fourfold pattern can be explained by the priority heuristic because of the following mechanism: in different choice problems, different attributes stop search.

Remark 5 In Result 4, a probability $p$ is considered to be low whenever $p \leq 0.1$ and in Result 5, a probability is considered to be low whenever $p \leq p^{*}$. Note that $p^{*}=0.1$ when $y=0$.

Remark 6 If one stays within the EUT framework and retains assumptions 1-3, the fourfold pattern can be explained by using a probability weighting function. For example, Prelec (1998) used the probability weighting function $w(p)=\exp (-(-\ln$ $p)^{a}$ ). In recent years, some researchers have elevated probability weighting to the status of a self-standing empirical phenomenon. This appears to have been established mostly by thought experiments: "Is it not clear that an increase from 0.01 to 0.02 in the probability of winning a prize means much more than an increase from 0.10 to 0.11 ?" We are not aware of direct evidence; rather the empirical data requires nonlinear probability weighting functions if one wants to retain assumptions 2 and 3. In contrast, Results 1 to 5 show that weighting of either probabilities or values is not necessary for analytically deriving major deviations from EUT. As mentioned above, this analytical result is confirmed by experimental evidence (Brandstätter et al. 2006). Across a total of 710 monetary choice problems, two striking results were obtained. When the ratio of expected values was between 1 and 2 (difficult problems), the priority heuristic predicted majority choice better than modifications of EUT, including cumulative prospect theory and heuristics such as minimax. When the ratio was larger (easy problems), the classical theory of expected value predicted best. Models that relied on nonlinear weighting of probabilities, such as cumulative prospect theory, were in each case second-best 
to either priority or expected value. Neither the heuristic nor the theory of expected value uses nonlinear weighting. 5

Our results are compatible with the interpretation of the priority heuristic as a "tiebreaking rule" which applies to situations where the choice options look similar and no-conflict solutions such as dominance and Rubinstein's similarity rule (see Section 5) cannot be found (Brandstätter et al. 2006; Erev et al. 2008). The famous anomalies emerge in exactly these cases.

In summary, in this section we have shown that the priority heuristic implies common consequence effects, common ratio effects, reflection effects, and the fourfold pattern of risk attitude (people are risk averse for gains if probabilities are high and for losses if probabilities are low, and risk taking for gains if probabilities are low and for losses if probabilities are high). Note that because the priority heuristic has no free parameters, it is also true that the heuristic predicts these phenomena simultaneously.

We want to emphasize that it is a major strength of a model of risky choice to predict these phenomena rather than simply being potentially consistent with the phenomena. Viscusi (1989) has made the same point when he showed that his prospective reference theory predicts, rather than assumes, violations of EUT such as the Allais paradox and the over-weighting of low-probability events. For example, he derived the key property $P(p) / P(q) \leq P(a p) / P(a q)$ (for $p<q$ and $a<1$ ) of the probability weighting function of prospect theory (Viscusi 1989, pp. 249-250).

\section{What preferences does the priority heuristic represent?}

The priority heuristic does not assume preferences on the space of gambles - it is a model for the process of comparison between two gambles. It is possible, however, to study which axioms the preferences, represented by the heuristic, satisfy. EUT is based on the axioms of independence and transitivity, together with the more technical axioms of completeness and continuity. We have already shown that independence $^{6}$ is not satisfied by the heuristic, consistent with experimental

\footnotetext{
${ }^{5}$ Peter Todd (December 2003, personal communication) suggested a way of connecting the priority heuristic to probability weighting. If one takes the one-tenth aspiration level for probabilities of the heuristic and makes the auxiliary assumption that a "subjective" probability is "weighted" to be the arithmetic mean of all probabilities from which it differs by less than 0.1 , one gets what appears as underweighting and overweighting at the ends of the probability scale: For $p<0.1$, the subjective probabilities are larger than the objective probabilities, which is the same effect as overweighting. For instance, the objective probability 0.05 differs by not more than 0.1 from all probabilities in the interval [0, $0.15]$, so the subjective probability would be estimated as 0.075 . Similarly, for $p>0.9$, the subjective probabilities are smaller than the objective probabilities, which corresponds to underweighting. If $p$ is in $[0.1,0.9]$, there is no difference between objective and subjective probabilities.

${ }^{6}$ There are many conceptualizations of independence (Marley and Luce 2005). It is easy to see that the priority heuristic satisfies some of them such as branch independence (Marley and Luce 2005, p. 98) and not others such as co-monotonic consequence monotonicity (Marley and Luce 2005, p. 79), but we do not go into these details here.
} 
evidence. Does the heuristic allow for occasional intransitive preferences? The heuristic represents transitive (TR) preferences if the following holds:

$$
\text { For all } G>_{h} G^{\prime} \text { and } G^{\prime}>_{h} G^{\prime \prime} \text {, it holds that } G>_{h} G^{\prime \prime} \text {. }
$$

(TR) would hold if the priority heuristic would evaluate options independently and assign a single numerical value to them. The heuristic, however, evaluates options relative to each other and can predict occasional intransitivities. Take, for example, $G=(3,0.49 ; 0,0.51), G^{\prime}=(2,0.55 ; 0,0.45)$, and $G^{\prime \prime}=(1,0.6 ; 0,0.4)$. Then, by maximum gain, $G>{ }_{h} G^{\prime}$ and $G^{\prime}>_{h} G^{\prime \prime}$, but, by probability of minimum gain, $G^{\prime \prime}>_{h}$ $G$. More generally, the following holds:

Result 6 (transitivity) Let $G, G^{\prime}$, and $G^{\prime \prime}$ be gambles with one zero and one non-zero outcome. (i) If $G>{ }_{h} G^{\prime}$ and $G^{\prime}>_{h} G^{\prime \prime}$ both hold by probability of minimum gain, (TR) holds. In the other cases, it is possible that (TR) does not hold. (ii) If $G>_{h} G^{\prime}$ and $G^{\prime}>_{h}$ $G^{\prime \prime}$ both hold by probability of minimum loss, (TR) holds. In the other cases, it is possible that (TR) does not hold.

Proof (1) For this proof, let $G=(x, p ; 0,1-p), G^{\prime}=\left(x^{\prime}, p^{\prime} ; 0,1-p^{\prime}\right)$, and $G^{\prime \prime}=\left(x^{\prime \prime}, p^{\prime \prime}\right.$; $\left.0,1-p^{\prime \prime}\right)$. We assume that $G>{ }_{h} G^{\prime}$ and $G^{\prime}>_{h} G^{\prime \prime}$.

If $G>{ }_{h} G^{\prime}$ and $G^{\prime}>{ }_{h} G^{\prime}$ both hold by probability of minimum gain, it has to be that $G>{ }_{h} G^{\prime \prime}$ because $p-p^{\prime}>0.1$ and $p^{\prime}-p^{\prime \prime}>0.1$ imply $p-p^{\prime \prime}>0.1$.

Next we show that for the following three cases it can be that $G^{\prime \prime}>{ }_{h} G$.

If $G>{ }_{h} G^{\prime}$ by probability of minimum gain and $G^{\prime}>{ }_{h} G^{\prime \prime}$ by maximum gain, it holds that $p-p^{\prime}>0.1,\left|p^{\prime}-p^{\prime \prime}\right| \leq 0.1$, and $x^{\prime}>x^{\prime \prime}$. These do not exclude $\left|p-p^{\prime \prime}\right| \leq$ 0.1 and $x^{\prime \prime}>x$ which means that $G^{\prime \prime}>{ }_{h} G$ is possible (e.g., take $x=1, x^{\prime}=3, x^{\prime \prime}=2, p=$ $0.51, p^{\prime}=0.4$, and $p^{\prime \prime}=0.45$ ).

If $G>{ }_{h} G^{\prime}$ by maximum gain and $G^{\prime}>{ }_{h} G^{\prime \prime}$ by probability of minimum gain, it holds that $\left|p-p^{\prime}\right| \leq 0.1, x>x^{\prime}$, and $\left|p-p^{\prime \prime}\right|>0.1$. It can again be that $G^{\prime \prime}>{ }_{h} G$ because it is again possible that $\left|p-p^{\prime \prime}\right| \leq 0.1$ and $x^{\prime \prime}>x$ (take, e.g., $x=2, x^{\prime}=1, x^{\prime \prime}=$ $\left.3, p=0.45, p^{\prime}=0.51, p^{\prime \prime}=0.4\right)^{7}$

The last case, where $G>{ }_{h} G^{\prime}$ and $G^{\prime}>_{h} G^{\prime \prime}$ both hold by maximum gain, also does not exclude that $G^{\prime \prime}>{ }_{h} G$. This is illustrated by the example preceding the result.

(2) It suffices to combine (i) with Result 3.

Remark 7 Note that, according to the priority heuristic, the psychological reason for intransitivity is a threshold phenomenon, well known from psychophysics: when two differences (in the last case in the proof of Result 6 in probabilities of minimum gain), each below the aspiration level (in that case, 0.06 and 0.05 ) are combined, the result $(0.11)$ can exceed the aspiration level, and this may result in an intransitivity. Note that such intransitivity due to thresholds should not be seen as a form of irrationality. Our sensory systems, visual or tactile, rely on such thresholds and produce intransitivity in rare occasions but that does not mean that these systems are

\footnotetext{
${ }^{7}$ Note that the counterexamples for these two cases are formally identical: in the second case, $G^{\prime}$ plays the role that $G$ played in the first case, $G^{\prime \prime}$ plays the role that $G^{\prime}$ played in the first case, and $G$ plays the role that $G^{\prime \prime}$ played in the first case.
}

Springer 
inadequate. Every intelligent system produces systematic errors in an uncertain world (Gigerenzer 2005). Note that others have also argued that transitivity may not be normatively compelling (Fishburn 1991) or, more generally, have discussed the potential value of inconsistency (Engel and Daston 2006).

The result above can be extended as follows:

Result 7 (transitivity) Let $G, G^{\prime}$, and $G^{\prime \prime}$ be gambles with two non-zero outcomes. (i) If $G>{ }_{h} G^{\prime}$ and $G^{\prime}>_{h} G^{\prime \prime}$ both hold by minimum gain or by probability of minimum gain, (TR) holds. In the other cases, it is possible that (TR) does not hold. (ii) If $G>_{h}$ $G^{\prime}$ and $G^{\prime}>{ }_{h} G^{\prime \prime}$ both hold by minimum loss or by probability of minimum loss, (TR) holds. In the other cases, it is possible that (TR) does not hold.

Proof In addition to the four cases that are consistent with $G>{ }_{h} G^{\prime}$ and $G^{\prime}>{ }_{h} G^{\prime \prime}$ for gambles with one non-zero outcome, there are five more to be considered for gambles with two non-zero outcomes: (a) $G>_{h} G^{\prime}$ and $G^{\prime}>_{h} G^{\prime \prime}$ both hold by minimum gain, (b) $G>{ }_{h} G^{\prime}$ holds by minimum gain and $G^{\prime}>_{h} G^{\prime \prime}$ holds by probability of minimum gain, (c) $G>{ }_{h} G^{\prime}$ holds by minimum gain and $G^{\prime}>_{h} G^{\prime \prime}$ holds by maximum gain, (d) $G>{ }_{h} G^{\prime}$ holds by probability of minimum gain and $G^{\prime}>{ }_{h} G^{\prime \prime}$ holds by minimum gain, and (e) $G>{ }_{h} G^{\prime}$ holds by maximum gain and $G^{\prime}>_{h} G^{\prime \prime}$ holds by minimum gain.

For this proof, let $G=(x, p ; y, 1-p), G^{\prime}=\left(x^{\prime}, p^{\prime} ; y^{\prime}, 1-p^{\prime}\right)$, and $G^{\prime \prime}=\left(x^{\prime \prime}, p^{\prime \prime} ; y^{\prime \prime}, 1-p^{\prime \prime}\right)$ with $x>y, x^{\prime}>y^{\prime}$, and $x^{\prime \prime}>y^{\prime \prime}$.

In (a), it holds that $y-y^{\prime}>(0.1) \max \left\{x, x^{\prime}\right\}$ and $y^{\prime}-y^{\prime \prime}>(0.1) \max \left\{x^{\prime}, x^{\prime \prime}\right\}$, and thus also $y-y^{\prime \prime}>(0.1)\left(\max \left\{x, x^{\prime}\right\}+\max \left\{x^{\prime}, x^{\prime \prime}\right\}\right)$. Because $\max \left\{x, x^{\prime}\right\}+\max \left\{x^{\prime}\right.$, $\left.x^{\prime \prime}\right\}>\max \left\{x, x^{\prime}, x^{\prime \prime}\right\}$, it also holds that $G>_{h} G^{\prime \prime}$.

In (b), it holds that $y-y^{\prime}>(0.1) \max \left\{x, x^{\prime}\right\},\left|y^{\prime}-y^{\prime \prime}\right| \leq(0.1) \max \left\{x^{\prime}, x^{\prime \prime}\right\}$, and $p^{\prime}-p^{\prime \prime}>0.1$. These do not exclude $\left|y-y^{\prime \prime}\right| \leq(0.1) \max \left\{x, x^{\prime \prime}\right\},\left|p-p^{\prime \prime}\right| \leq 0.1$, and $x^{\prime \prime}>x$, which means that $G^{\prime \prime}>{ }_{h} G$ is possible: for example, take $x=5, x^{\prime}=6, x^{\prime \prime}=11, p=$ $0.4, p^{\prime}=0.6, p^{\prime \prime}=0.45, y=3, y^{\prime}=1$, and $y^{\prime \prime}=2$.

In (c), it holds that $y-y^{\prime}>(0.1) \max \left\{x, x^{\prime}\right\},\left|y^{\prime}-y^{\prime \prime}\right| \leq(0.1) \max \left\{x^{\prime}, x^{\prime \prime}\right\}$, $\left|p^{\prime}-p^{\prime \prime}\right| \leq 0.1$, and $x^{\prime}>x^{\prime \prime}$. These do not exclude $\left|y-y^{\prime \prime}\right| \leq(0.1) \max \left\{x, x^{\prime \prime}\right\}$, and $p^{\prime \prime}-p>0.1$, which means that $G^{\prime \prime}>{ }_{h} G$ is possible: for example, take $x=11, x^{\prime}=6, x^{\prime \prime}=$ $5, p=0.4, p^{\prime}=0.55, p^{\prime \prime}=0.6, y=3, y^{\prime}=1$, and $y^{\prime \prime}=2$.

In (d), it holds that $\left|y-y^{\prime}\right| \leq(0.1) \max \left\{x, x^{\prime}\right\}, p-p^{\prime}>0.1$, and $y^{\prime}-y^{\prime \prime}>(0.1)$ $\max \left\{x^{\prime}, x^{\prime \prime}\right\}$. These do not exclude $\left|y-y^{\prime \prime}\right| \leq(0.1) \max \left\{x, x^{\prime \prime}\right\},\left|p^{\prime \prime}-p\right| \leq 0.1$, and $x^{\prime \prime}>x$, which means that $G^{\prime \prime}>{ }_{h} G$ is possible: for example, take $x=11, x^{\prime}=5, x^{\prime \prime}=6, p=$ $0.6, p^{\prime}=0.4, p^{\prime \prime}=0.55, y=2, y^{\prime}=3$, and $y^{\prime \prime}=1 .{ }^{8}$

In (e), it holds that $\left|y-y^{\prime}\right| \leq(0.1) \max \left\{x, x^{\prime}\right\},\left|p-p^{\prime}\right| \leq 0.1, x>x^{\prime}$, and $y^{\prime}-y^{\prime \prime}>$ (0.1) $\max \left\{x^{\prime}, x^{\prime \prime}\right\}$. These do not exclude $\left|y-y^{\prime \prime}\right| \leq(0.1) \max \left\{x, x^{\prime \prime}\right\}$ and $p^{\prime \prime}-p>0.1$, which means that $G^{\prime \prime}>{ }_{h} G$ is possible: for example, take $x=11, x^{\prime}=5, x^{\prime \prime}=6, p=0.45$, $p^{\prime}=0.4, p^{\prime \prime}=0.6, y=2, y^{\prime}=3$, and $y^{\prime \prime}=1$.

(2) It suffices to combine (i) with Result 3.

\footnotetext{
${ }^{8}$ Note that, as in the proof of Result 6, the counterexamples for the cases (c) and (d) are formally identical. This is also true for the counterexamples for the cases (b) and (e).
} 
The independence axiom is often weakened to betweenness. Another commonly used axiom is monotonicity. In the remainder of this section, we examine whether the preferences represented by the priority heuristic satisfy completeness (CM), continuity $(\mathrm{CN})$, betweenness $(\mathrm{BE})$, and monotonicity $(\mathrm{MO})$. That is, we check whether the following statements hold:

For all $G, G^{\prime}$, it holds that $G>_{h} G^{\prime}$ or $G^{\prime}>_{h} G$ or $G={ }_{h} G^{\prime}$.

For all $G>_{h} G^{\prime}>_{h} G^{\prime} \prime$, there exists $0<p<1: G^{\prime}={ }_{h}\left(G, p ; G^{\prime \prime}, 1-p\right)$.

For all $G>_{h} G^{\prime}$ and $0<p<1$, it holds that $G>_{h}\left(G, p ; G^{\prime}, 1-p\right)>_{h} G^{\prime}$.

If, for $i=1, \ldots, n-1, \sum_{j=1, \ldots, i} p_{j} \geq \sum_{j=1, \ldots, i} q_{j}$ and $x_{1} \geq \ldots \geq x_{n}$,

$$
\text { then }\left(x_{1}, p_{i} ; \ldots ; x_{n}, p_{n}\right) \geq_{h}\left(x_{1}, q_{1} ; \ldots ; x_{n}, q_{n}\right) \text {. }
$$

Result 8 (other axioms) Statements (CM) and (MO) hold. Statements (CN) and (BE) do not.

Proof By the definition of the relation " $>h$ ", $(\mathrm{CM})$ holds.

For (MO) to hold it suffices to show that (a) $p_{1} \geq q_{1}$ and (b) $p_{n} \leq q_{n}$. To show (a), we set $i=1$ in the condition $\sum_{j=1, \ldots, i} p_{j} \geq \sum_{j=1, \ldots, i} q_{j}$. To show (b), we set $i=n-1$ in the condition $\sum_{j=1, \ldots, i} p_{j} \geq \sum_{j=1, \ldots, i} q_{j}$ (and also use that $\sum_{j=1, \ldots, n} p_{j}=$ $\left.\sum_{j=1, \ldots, n} q_{j}=1\right)$.

To show that $(\mathrm{CN})$ does not hold, we construct the following counterexample: Take $G=(10,0.8 ; 0,0.2), G^{\prime}=(5,0.6 ; 0,0.4)$ and $G^{\prime \prime}=(10,0.4 ; 0,0.6)$. By the probability of minimum gain, $G>{ }_{h} G^{\prime}>{ }_{h} G^{\prime \prime}$. For an arbitrary $p$, the reduced form of $\left(G, p ; G^{\prime \prime}, 1-p\right)$ is $(10,(0.8) p+0.4(1-p) ; 0,(0.2) p+0.6(1-p))$. But it is impossible that $(10,(0.8) p+0.4(1-p) ; 0,(0.2) p+0.6(1-p))={ }_{h}(5,0.6 ; 0,0.4)$ because maximum gains are unequal.

To show that (BE) does not hold, we construct a counterexample where $G>_{h} G^{\prime}$ and $0<p<1$ but it does not hold that $G>_{h}\left(G, p ; G^{\prime}, 1-p\right)$ : Take $G=(5,0.6 ; 1,0.4)$, $G^{\prime}=(20,0.4 ; 2,0.6)$ and $p=0.9$. By the probability of minimum gain, $G>_{h} G^{\prime}$. The reduced form of $\left(G, p ; G^{\prime}, 1-p\right)$ is $(20,0.04 ; 5,0.54 ; 2,0.06 ; 1,0.36)$. By the maximum gain, $(20,0.04 ; 5,0.54 ; 2,0.06 ; 1,0.36)>_{h}(5,0.6 ; 1,0.4)$.

Remark 8 As seen in (BE), given that $G>{ }_{h} G^{\prime}$ and $0<p<1$, the betweenness axiom makes two requests (see also Camerer and Ho 1994): $G>_{h}\left(G, p ; G^{\prime}, 1-p\right.$ ) (called quasi-convexity) and $\left(G, p ; G^{\prime}, 1-p\right)>{ }_{h} G^{\prime}$ (called quasi-concavity). Because the priority heuristic predicts reflection effects, it is easy to see that the counterexample to quasi-convexity, that used gains, immediately suggests a counterexample to quasiconcavity, that uses losses, that is, $G=(-20,0.4 ;-2,0.6), G^{\prime}=(-5,0.6 ;-1,0.4)$ and $p=0.1$. It turns out, however, that the non-strict version of quasi-convexity holds for losses and that the non-strict version of quasi-concavity holds for gains. 
Result 9 (non-strict quasi-concavity) For all gambles $G>_{h} G^{\prime}$ with gains and for $0<$ $p<1$, it holds that $\left(G, p ; G^{\prime}, 1-p\right) \geq_{h} G^{\prime}$.

Proof We show that, given gambles $G>_{h} G^{\prime}$ with gains and $0<p<1, G^{\prime}>_{h}\left(G, p ; G^{\prime}\right.$, $1-p)$ is impossible. To begin with, it is obvious that $G^{\prime}>_{h}\left(G, p ; G^{\prime}, 1-p\right)$ cannot hold by maximum gain.

For this proof, let $x_{G}$ and $p_{G}$ be, respectively, the minimum gain of $G$ and its probability. Also, let $x_{G}{ }^{\prime}$ and $p_{G}{ }^{\prime}$ be, respectively, the minimum gain of $G^{\prime}$ and its probability.

Minimum gain cannot stop search and imply $G^{\prime}>_{h}\left(G, p ; G^{\prime}, 1-p\right)$ because then it would also hold that $x_{G}{ }^{\prime}>x_{G}$ and that $x_{G}{ }^{\prime}-x_{G}$ exceeds the aspiration level for gains which imply that $G^{\prime}>{ }_{h} G$.

For the probability of minimum gain, we distinguish three cases: (a) $x_{G}{ }^{\prime}=x_{G}$, (b) $x_{G}{ }^{\prime}<x_{G}$, and (c) $x_{G}<x_{G}{ }^{\prime}$. For (a), (b) and (c) we will show that the probability of minimum gain does not stop search or that it cannot imply $G^{\prime}>_{h}\left(G, p ; G^{\prime}, 1-p\right)$.

If (a) holds, the difference between the probabilities of minimum gains of $G^{\prime}$ and $\left(G, p ; G^{\prime}, 1-p\right)$ equals $p p_{G}+(1-p) p_{G}{ }^{\prime}-p_{G}{ }^{\prime}=p\left(p_{G}-p_{G}{ }^{\prime}\right)<p_{G}-p_{G^{\prime}} \leq 0.1$, where the last inequality holds because $p_{G}-p_{G^{\prime}}>0.1$ would, together with (a), imply $G^{\prime}>_{h} G$.

If (b) holds, $\left(G, p ; G^{\prime}, 1-p\right)$ is more attractive in probability of minimum gain than $G^{\prime}$ (because $(1-\mathrm{p}) p_{G^{\prime}}<p_{G^{\prime}}$ ) and thus it cannot be that $G^{\prime}>_{h}\left(G, p ; G^{\prime}, 1-p\right)$.

If (c) holds, we only need to consider the case where $G^{\prime}$ is more attractive in probability of minimum gain than $\left(G, p ; G^{\prime}, 1-p\right)$. Then the difference between these probabilities equals $p p_{G}-p_{G}^{\prime}$. For this to exceed the aspiration level for probabilities, it must be that $p>\left(0.1+p_{G}{ }^{\prime}\right) / p_{G}$. Because $1>p$, this implies that $p_{G^{-}}$ $p_{G}{ }^{\prime}>0.1$. But $p_{G}-p_{G}{ }^{\prime}>0.1$, together with (c), implies $G^{\prime}>{ }_{h} G$.

Remark 9 The strict version of Result 9 does not hold: As a counterexample take $G=(10,0.5 ; 0,0.5), G^{\prime}=(10,0.34 ; 0,0.66)$ and $p=0.5$.

Remark 10 Quasi-concave preferences indicate a preference for randomization: a mixture of equally desirable gambles is preferred to any of the gambles (Camerer and Ho 1994, p. 173). Graphically, quasi-concave preferences are represented by convex indifference curves in the Marchak-Machina triangle.

Combining Result 9 and Result 3, yields the following:

Result 10 (non-strict quasi-convexity) For all gambles $G>{ }_{h} G^{\prime}$ with losses and for $0<$ $p<1$, it holds that $G \geq_{h}\left(G, p ; G^{\prime}, 1-p\right)$.

In summary, the priority heuristic represents preferences that are complete, monotone, and (non-strictly) quasi-concave for gains and (non-strictly) quasiconvex for losses and are, under some conditions, transitive. The preferences do not embody continuity and betweeness. More generally, instead of being based on axioms, the priority heuristic models choice by incorporating psychological principles: relative evaluation, search stopped by aspiration levels, and avoiding trade-offs. These psychological principles underlie our conception of bounded rationality. Other researchers have modeled bounded rationality in different but related ways. 


\section{Relation to Rubinstein's (1988) similarity model}

Rubinstein's (1988) model presupposes two similarity relations, one in the space of values and one in the space of probabilities; both are defined by six axioms. These relations play a similar role to the aspiration levels of the priority heuristic. For example, the relation " $\sim$ " that is defined by $p \sim p^{\prime}$ if and only if $\left|p-p^{\prime}\right| \leq 0.1$ satisfies the six axioms (p. 148). The two relations can be seen as free parameters; in fact, for any relation " $\sim$ " and for any scalar $k>1$, there exist a strictly increasing and positive function $H$ on the unit interval so that $x \sim y$ if and only if $1 / k \leq H(x) / H(y) \leq k$ (p. 149).

Rubinstein models the choice between $(x, p ; 0,1-p)$ and $\left(y, p^{\prime} ; 0,1-p^{\prime}\right)$ where $0<x$, $p, y, p^{\prime}<1$. We write $x \sim y$ to denote that the two gains $x$ and $y$ are similar and $p \sim p^{\prime}$ to denote that the two probabilities $p$ and $p^{\prime}$ are similar. In the first step of the model it is asked if $x>y$ and $p>p^{\prime}$. If yes, $(x, p ; 0,1-p)$ is chosen. If not, in the second step, it is asked if one of the conditions $\left\{p \sim p^{\prime}\right.$ and not $(x \sim y)$ and $\left.x>y\right\}$ or $\left\{x \sim y\right.$ and not $\left(p \sim p^{\prime}\right)$ and $\left.p>p^{\prime}\right\}$ holds. If yes, $(x, p ; 0,1-p)$ is chosen. If not, the model makes no prediction. For extensions, see Aizpurua et al. (1993), Leland (1994) and Buschena and Zilberman (1995, 1999).

Like the priority heuristic, the similarity model attempts to describe the process of choice and does not transform values and probabilities. However, it differs in so far as it does not employ limited search (except for the dominance check) and is not lexicographic because it does not specify an order in which values and probabilities are considered. Furthermore, it has an EUT representation (Rubinstein 1988, pp. 150-151) and in this sense it implements independent evaluations that make trade-offs. Note that other similarity models do not have EUT representations (Leland 1994). Finally, the predictions of the similarity model can be tuned because it has adjustable parameters.

Can Rubinstein's model reproduce the predictions of the priority heuristic? In the simple case of dominance, i.e., $x>y$ and $p>p^{\prime}$, the two models make identical predictions. Can the parameters of the similarity model be set so that it reproduces the predictions of the priority heuristic in cases of conflict, that is, $x>y$ and $p<p^{\prime}$, as well? This is not the case: For example, assume $\left|p-p^{\prime}\right| \leq 0.1$; the priority heuristic then predicts that $(x, p ; 0,1-p)$ is chosen. To match this for all $x$ and $y$, the similarity relation in the space of maximum gains needs to be such that for all $x$ and $y$ with $x>y$ it does not hold that $x \sim y$. But this means that any maximum gain would not be similar to any different maximum gain, which contradicts the axiom of nondegeneracy (Rubinstein 1988, p. 148).

In summary, the similarity model keeps assumptions (4) and (5) of EVT, as the priority heuristic does. On the other hand, the similarity model does not employ limited search, is not lexicographic and uses free parameters. Consistent with these conceptual differences, the predictions of the two models differ. In fact, the two models can be seen as complementary in the sense that the priority heuristic is a candidate for the third, unspecified step of the similarity model, when the choice is "difficult".

\section{Bounded rationality}

The concept of bounded rationality has been often defined as optimization under constraints, such as information and deliberation costs (Conlisk 1996; Sargent 1993). 
Yet it also has been understood in a different way, in terms of a "map" of cognitive illusions (or anomalies), as in Kahneman (2003). Note that the first involves optimization and emphasizes rationality whereas the second does neither and instead emphasizes irrationality.

The priority heuristic does not involve computing an optimum but deals with limited information and time by using simple stopping rules that make it fast and frugal. But the heuristic is not an instance of cognitive illusions either. There exists a third interpretation of bounded rationality. We see the priority heuristic in the bounded rationality framework proposed by Gigerenzer and Selten (2001): modeling how people actually make decisions based on an "adaptive toolbox" of heuristics that are fast and frugal without optimization computations.

The priority heuristic is a member of a class of models known as one-reason decision-making (Gigerenzer 2004), which also includes fast and frugal trees (Martignon et al. 2003) and Take The Best (Gigerenzer and Goldstein 1996). These models implement limited search with stopping rules (in contrast to assumption 2), make no trade-offs (in contrast to assumption 3), do not transform the information given (following assumptions 4 and 5), and some assume that options are evaluated relatively (while others model independent evaluations as in assumption 1).

Since the codification of rational choice in the seventeenth century in terms of the maximization of the expected value, the dominant strand for dealing with discrepancies between theory and evidence has been the gradual transformation of the values and probabilities in the equation. For example, Daniel Bernoulli argued that rich men valued increases in their wealth less than poor men did and proposed that the utility of money is a logarithmic function. ${ }^{9}$ The more general characteristics - that options are considered independently, that all pieces of information are used and that these are weighted and added-have remained largely unchallenged.

In this paper, we showed that a simple heuristic using relative evaluation, limited search, and no trade-offs implies common consequence effects, common ratio effects, reflection effects and the fourfold pattern of risk attitude (people are risk-averse for gains if probabilities are high, risk-taking for gains if probabilities are low (as in buying lottery tickets), risk-averse for losses when probabilities are low (as in buying insurance policies), and risk-taking for losses when probabilities are high). Because the heuristic has no free parameters, it predicts these phenomena simultaneously, rather than being potentially consistent with the phenomena. We also examined the kind of preferences represented by the heuristic and found conditions under which it obeys axioms such as transitivity, betweenness, and monotonicity. These analytical results, combined with the experimental results reviewed, contribute to the objective of constructing a descriptive theory of decision under risk in the spirit of bounded rationality.

\footnotetext{
${ }^{9}$ Note that he has been recently accused of committing an error in his analysis: "Bernoulli's model of utility is flawed because it is reference independent: It assumes that the utility that is assigned to a given state of wealth does not vary with the decision maker's initial state of wealth" (Kahneman 2003, p. 704). He continues, "I call this Bernoulli's error". We would like to vindicate Bernoulli from the charge. Contrary to Kahneman's assertion, Bernoulli was explicit about the impact of initial state of wealth on utility: "Thus there is no doubt that a gain of one thousand ducats is more significant to a pauper than to a rich man though both gain the same amount"(Bernoulli (1738, p. 24). And Bernoulli explicitly included initial wealth in his equations and diagrams: “... let $\mathrm{AB}$ represent the quantity of goods initially possessed.” (p. 26). For general treatments of Bernoulli, see Daston (1988, pp. 70-77) and Jorland (1987).
} 


\section{References}

Albers, W. (2001). Prominence theory as a tool to model boundedly rational decisions. In G. Gigerenzer, \& R. Selten (Eds.), Bounded rationality: The adaptive toolbox. Cambridge, MA: MIT Press.

Allais, M. (1979). Foundations of a positive theory of choice involving risk, and a criticism of the postulates and axioms of the American School. In M. Allais, \& O. Hagen (Eds.), Expected utility hypothesis and the Allais paradox. Dordrecht: D. Reidel Publishing Co. (original work published 1952).

Aizpurua, J. M., Ichiishi, T., Nieto, J., \& Uriarte, J. R. (1993). Similarity and preferences in the space of simple lotteries. Journal of Risk and Uncertainty, 6(3), 289-297.

Baucells, M., Carrasco, J. A., \& Hogarth, R. M. (2008). Cumulative dominance and heuristic performance in binary multi-attribute choice. Operations Research (in press).

Bernoulli, D. (1738). Exposition of a new theory on the measurement of risk. Comentarii Academiae Scientiarum Imperialis Petropolitanae. Translated and reprinted in Econometrica 22(1954), 23-36.

Birnbaum, M. (2004). Causes of Allais common consequence paradoxes: An experimental dissection. Journal of Mathematical Psychology, 48(2), 87-106.

Birnbaum, M., \& Chavez, A. (1997). Tests of theories of decision making: Violations of branch independence and distribution independence. Organizational Behavior and Human Decision Processes, 71(2), 161-194.

Blavatskyy, P. R. (2005). Back to the St. Petersburg paradox? Management Science, 51(4), 677-678.

Brandstätter, E., Gigerenzer, G., \& Hertwig, R. (2006). The priority heuristic: A process model of risky choice. Psychological Review, 113(2), 409-432.

Brighton, H. (2006). Robust inference with simple cognitive models. In C. Lebiere, \& R. Wray (Eds.), Between a rock and a hard place: Cognitive science principles meet AI-hard problems. Papers from the AAAI spring symposium (AAAI Tech. Rep. No. SS-06-03). Menlo Park, CA: AAAI Press.

Bröder, A. (2000). Assessing the empirical validity of the 'take-the-best' heuristic as a model of human probabilistic inference. Journal of Experimental Psychology. Learning, Memory, and Cognition, 26 (5), 1332-1346.

Bröder, A. (2003). Decision making with the 'adaptive toolbox': Influence of environmental structure, intelligence, and working memory load. Journal of Experimental Psychology, 29(4), 611-625.

Bröder, A., \& Schiffer, S. (2003). Take the best versus simultaneous feature matching: Probabilistic inferences from memory and effects of representation format. Journal of Experimental Psychology. General, 132(2), 277-293.

Bröder, A., \& Gaissmaier, W. (2007). Sequential processing of cues in memory-based multiattribute decisions. Psychonomic Bulletin and Review, 14(5), 895-900.

Buschena, D., \& Zilberman, D. (1995). Performance of the similarity hypothesis relative to existing models of risky choice. Journal of Risk and Uncertainty, 11(3), 233-262.

Buschena, D., \& Zilberman, D. (1999). Testing the effects of similarity on risky choice: Implications for violations of expected utility. Theory and Decision, 46(3), 251-276.

Camerer, C., \& Ho, T.-H. (1994). Violations of the betweenness axiom and nonlinearity in probability. Journal of Risk and Uncertainty, 8(2), 167-196.

Conlisk, J. (1996). Why bounded rationality? Journal of Economic Literature, 34(2), 669-700.

Daston, L. J. (1988). Classical probability in the enlightenment. Princeton, NJ: Princeton University Press.

Engel, C., \& Daston, L. J. (Eds). (2006). Is there value in inconsistency? Baden-Baden: Nomos.

Erev, I., Roth, A. E., Slonim, R. L., \& Barron, G. (2002). Combining a theoretical prediction with experimental evidence to yield a new prediction: an experimental design with a random sample of tasks. Unpublished manuscript, Columbia University and Faculty of Industrial Engineering and Management, Technion, Haifa, Israel.

Erev, I., Glozman, I., \& Hertwig, R. (2008). What impacts the impact of rare events. Journal of Risk and Uncertainty, 36(2), 153-177.

Fishburn, P. C. (1974). Lexicographic orders, utilities and decision rules: A survey. Management Science, 20(11), 1442-1471.

Fishburn, P. C. (1991). Nontransitive preferences in decision theory. Journal of Risk and Uncertainty, 4, $113-134$.

Ford, J. K., Schmitt, N., Schechtman, S. L., Hults, B. H., \& Doherty, M. L. (1989). Process tracing methods: Contributions, problems, and neglected research questions. Organizational Behavior and Human Decision Processes, 43(1), 75-117. 
Gigerenzer, G. (2004). Fast and frugal heuristics: The tools of bounded rationality. In D. Koehler, \& N. Harvey (Eds), Blackwell handbook of judgment and decision making (pp. 62-88). Malden: Blackwell.

Gigerenzer, G. (2005). I think, therefore I err. Social Research, 72(1), 195-218.

Gigerenzer, G., \& Goldstein, D. G. (1996). Reasoning the fast and frugal way: Models of bounded rationality. Psychological Review, 103(4), 650-669.

Gigerenzer, G., \& Richter, H. R. (1990). Context effects and their interaction with development: area judgments. Cognitive Development, 5(3), 235-264.

Gigerenzer, G., \& Selten, R. (Eds). (2001). Bounded rationality: The adaptive toolbox. Cambridge, MA: MIT Press.

Gigerenzer, G., \& Todd, P. M. (1999). Simple heuristics that make us smart. New York: Oxford University Press.

Gilbride, T. J., \& Allenby, G. M. (2004). A choice model with conjunctive, disjunctive, and compensatory screening rules. Marketing Science, 23(3), 391-406.

Heckhausen, H. (1991). Motivation and action (Peter K. Leppmann, trans.). Berlin: Springer.

Hogarth, R. M., \& Karelaia, N. (2005). Simple models for multi-attribute choice with many alternatives: When it does and does not pay to face trade-offs with binary attributes. Management Science, 51(12), $1860-1872$.

Hogarth, R. M., \& Karelaia, N. (2006). 'Take-the-best' and other simple strategies: Why and when they work 'well' with binary cues. Theory and Decision, 61(3), 205-249.

Jedetski, J., Adelman, L., \& Yeo, C. (2002). How web site decision technology affects consumers. IEEE Internet Computing, 6(2), 72-79.

Jorland, G. (1987). The Saint Petersburg paradox 1713-1937. In L. Krüger, G. Gigerenzer, \& M. S. Morgan (Eds.), The Probabilistic revolution, vol. 1. Ideas in the sciences. Cambridge, MA: MIT Press.

Kahneman, D. (2003). A perspective on judgment and choice: Mapping bounded rationality. American Psychologist, 58(9), 697-720.

Kahneman, D., \& Tversky, A. (1979). Prospect theory: An analysis of decision under risk. Econometrica, 47(2), 263-291.

Katsikopoulos, K. V., \& Fasolo, B. (2006). New tools for decision analysts. IEEE Transactions on Systems, Man, and Cybernetics: Systems and Humans, 36(5), 960-967.

Katsikopoulos, K. V., \& Martignon, L. (2006). Naive heuristics for paired comparisons: Some results on their relative accuracy. Journal of Mathematical Psychology, 50(5), 488-494.

Leland, J. W. (1994). Generalized similarity judgments: An alternative explanation for choice anomalies. Journal of Risk and Uncertainty, 9(2), 151-172.

Leland, J. W. (2002). Similarity judgments and anomalies in intertemporal choice. Economic Inquiry, 40 (4), 574-581.

Lilly, G. (1994). Bounded rationality: A Simon-like explication. Journal of Economic Dynamics and Control, 18(1), 105-230.

Loewenstein, G. F., Weber, E. U., Hsee, C. K., \& Welch, N. (2001). Risk as a feeling. Psychological Bulletin, 127(2), 267-286.

Loomes, G., \& Sugden, R. (1987). Testing for regret and disappointment in choice under uncertainty. The Economic Journal, 97, 118-129.

Lopes, L. L. (1995). Algebra and process in the modeling of risky choice. In J. R. Busemeyer, R. Hastie, \& D. Medin (Eds.), Decision making from the perspective of cognitive psychology. New York: Academic Press.

Lopes, L. L., \& Oden, G. C. (1999). The role of aspiration level in risky choice: A comparison of cumulative prospect theory and SP/A theory. Journal of Mathematical Psychology, 43(2), 286-313.

Luce, R. D. (1956). Semiorders and a theory of utility discrimination. Econometrica, 24(2), 178-191.

Luce, R. D. (1990). Rational versus plausible accounting equivalences in preference judgments. Psychological Science, 1(4), 225-234.

Luce, R. D., \& von Winterfeldt, D. (1994). What common ground exists for descriptive, prescriptive, and normative utility theories? Management Science, 40(2), 263-279.

MacCrimmon, K. R. (1968). Descriptive and normative implications of the decision-theory postulate. In K. H. Borch, \& J. Mossin (Eds.), Risk and uncertainty. New York: St. Martin's Press.

Marley, A. A. J., \& Luce, R. D. (2005). Independence properties vis-à-vis several utility representations. Theory and Decision, 58(1), 77-143.

Martignon, L., \& Hoffrage, U. (2002). Fast, frugal and fit: Lexicographic heuristics for paired comparison. Theory and Decision, 52(1), 29-71. 
Martignon, L., Vitouch, O., Takezawa, M., \& Forster, M. R. (2003). Naive and yet enlightened: from natural frequencies to fast and frugal decision trees. In D. Hardman, \& L. Macchi (Eds.), Thinking: Psychological perspectives on reasoning, judgment and decision making. Chichester, UK: Wiley.

Neilson, W., \& Stowe, J. (2002). A further examination of cumulative prospect theory parameterizations. The Journal of Risk and Uncertainty, 24(1), 31-46.

Nosofsky, R. M., \& Bergert, B. F. (2007). Limitations of exemplar models for multi-attribute probabilistic inference. Journal of Experimental Psychology. Learning, Memory, and Cognition, 33(6), 999-1119.

Parducci, A. (1965). Category judgment: A range-frequency model. Psychological Review, 72(6), 407418.

Payne, J. W., Bettman, J. R., \& Johnson, E. J. (1993). The adaptive decision maker. Cambridge, UK: Cambridge University Press.

Prelec, D. (1998). The probability weighting function. Econometrica, 66(3), 497-527.

Rieskamp, J., \& Hoffrage, U. (1999). When do people use simple heuristics and how can we tell? In G. Gigerenzer, \& P. M. Todd (Eds.), the ABC Research Group. Simple heuristics that make us smart. New York: Oxford University Press.

Rieskamp, J., \& Otto, P. E. (2006). SSL: A theory of how people learn to select strategies. Journal of Experimental Psychology. General, 135(2), 207-236.

Roberts, S., \& Pashler, H. (2000). How persuasive is a good fit? A comment on theory testing. Psychological Review, 107(2), 358-367.

Rubinstein, A. (1988). Similarity and decision-making under risk (is there a utility theory resolution to the Allais-paradox?). Journal of Economic Theory, 46(1), 145-153.

Sargent, T. J. (1993). Bounded rationality in macroeconomics. New York: Oxford University Press.

Schwarz, N. (1999). Self-reports: How the questions shape the answers. American Psychologist, 54(2), 93-105.

Savage, L. J. (1954). The foundations of statistics. New York: Wiley.

Schwarz, N., Hippler, H.-J., Deutsch, B., \& Strack, F. (1985). Response categories: Effects on behavioral reports and comparative judgments. Public Opinion Quarterly, 49(3), 388-395.

Selten, R. (2001). What is bounded rationality? In G. Gigerenzer, \& R. Selten (Eds.), Bounded rationality: The adaptive toolbox. Cambridge, MA: MIT Press.

Shafir, E., Simonson, I., \& Tversky, A. (1993). Reason-based choice. Cognition, 49(1/2), 11-36.

Simon, H. A. (1955). A behavioral model of rational choice. Quarterly Journal of Economics, 69(1), 99118.

Simon, H. A. (1956). Rational choice and the structure of the environment. Psychological Review, 63(2), $129-138$.

Slovic, P., Griffin, D., \& Tversky, A. (1990). Compatibility effects in judgment and choice. In R. M. Hogarth (Ed.), Insights in decision making: A tribute to Hillel J. Einhorn. Chicago: The University of Chicago Press.

Starmer, C. (2000). Developments in non-expected utility theory: The hunt for a descriptive theory of choice under risk. Journal of Economic Literature, 38(2), 332-382.

Sunstein, C. R. (2003). Terrorism and probability neglect. Journal of Risk and Uncertainty, 26(2/3), 121136.

Stigler, G. J. (1961). The economics of information. Journal of Political Economy, 69(3), 213-225.

Tversky, A. (1969). Intransitivity of preferences. Psychological Review, 76(1), 31-48.

Tversky, A., \& Fox, C. R. (1995). Weighing risk and uncertainty. Psychological Review, 102(2), 269-283.

Tversky, A., \& Kahneman, D. (1992). Advances in prospect theory: Cumulative representation of uncertainty. Journal of Risk and Uncertainty, 5(4), 297-323.

Viscusi, W. K. (1989). Prospective reference theory: Toward an explanation of the paradoxes. Journal of Risk and Uncertainty, 2, 235-264.

Wu, G., \& Gonzalez, R. (1996). Curvature of the probability weighting function. Management Science, 42 (12), 1676-1690.

Yee, M., Hauser, J. R., Orlin, J. B., \& Dahan, E. (2007). Greedoid-based non-compensatory two-stage consideration-then-choice inference. Marketing Science, 26(4), 532-549. 